\title{
Super-Resolution Genome Mapping in Silicon
}

\section{Nanochannels}

\author{
Jonathan Jeffet ${ }^{\dagger}$, Asaf Kobo ${ }^{\dagger}$, Tianxiang Su ${ }^{*}$, Assaf Grunwald ${ }^{\dagger}$, Ori Green ${ }^{\dagger}$, Adam N. Nilsson ${ }^{\S}$, \\ Eli Eisenberg", Tobias Ambjörnsson ${ }^{\S}$, Fredrik Westerlund', Elmar Weinhold ${ }^{\ddagger}$, Doron Shabat", \\ Prashant K. Purohit \& Yuval Ebenstein ${ }^{*}+$ \\ $\dagger$ Raymond and Beverly Sackler Faculty of Exact Sciences, Tel Aviv University, Tel Aviv \\ 6997801, Israel. \\ $\ddagger$ Department of Mechanical Engineering and Applied Mechanics, University of Pennsylvania, \\ Philadelphia, Pennsylvania, USA.
}

$¥$ School of Engineering and Applied Sciences, Harvard University, Cambridge, MA 02138, USA.

$\S$ Department of Astronomy and Theoretical Physics, Lund University, Sweden.

|l Department of Biology and Biological Engineering, Chalmers University of Technology, Gothenburg, Sweden.

$£$ Institute of Organic Chemistry, RWTH Aachen University, Aachen, D-52056, Germany.

KEYWORDS: Nanochannels, Super-resolution, Single-molecule, Optical genome mapping, DNA labeling, Confined polymers. 
IgorPro analysis - Locations, localization error and intensity: Localization of fluorescent labels was conducted using the IgorPro Localizer extension, written and maintained by Peter Dedecker et al. ${ }^{1}$. We found this software package superior to other available code in its ability to automatically detect fluorescent signal in relatively noisy environments. In each frame all spots were localized using a 2D Gaussian fits to intensity. The parameters for the 2D Gaussian localization were picked according to best performance for each DNA molecule individually. The localization algorithm that gave the best results was "Smooth Sigma" with a sigma parameter of 1.3 pixels and a threshold parameter of 0.5-0.6. After localization, the localized positions were joined into tracks, so that each fluorescent spot was given a single track. The tracking parameters were set to 3 pixels of maximum jump distance between frames and 7 frames of maximum blinking time. The shortest tracks produced were 4 point tracks. If needed, tracks were combined to create single tracks for each fluorescent label according to the proximity between the shorter tracks. The output parameters for each of the fluorescent spots localized in the track consisted of: the frame number, integrated intensity, $\mathrm{X}$ location in pixels, $\mathrm{Y}$ location in pixels, $\mathrm{X}$ and $\mathrm{Y}$ localization errors and localization PSF. 


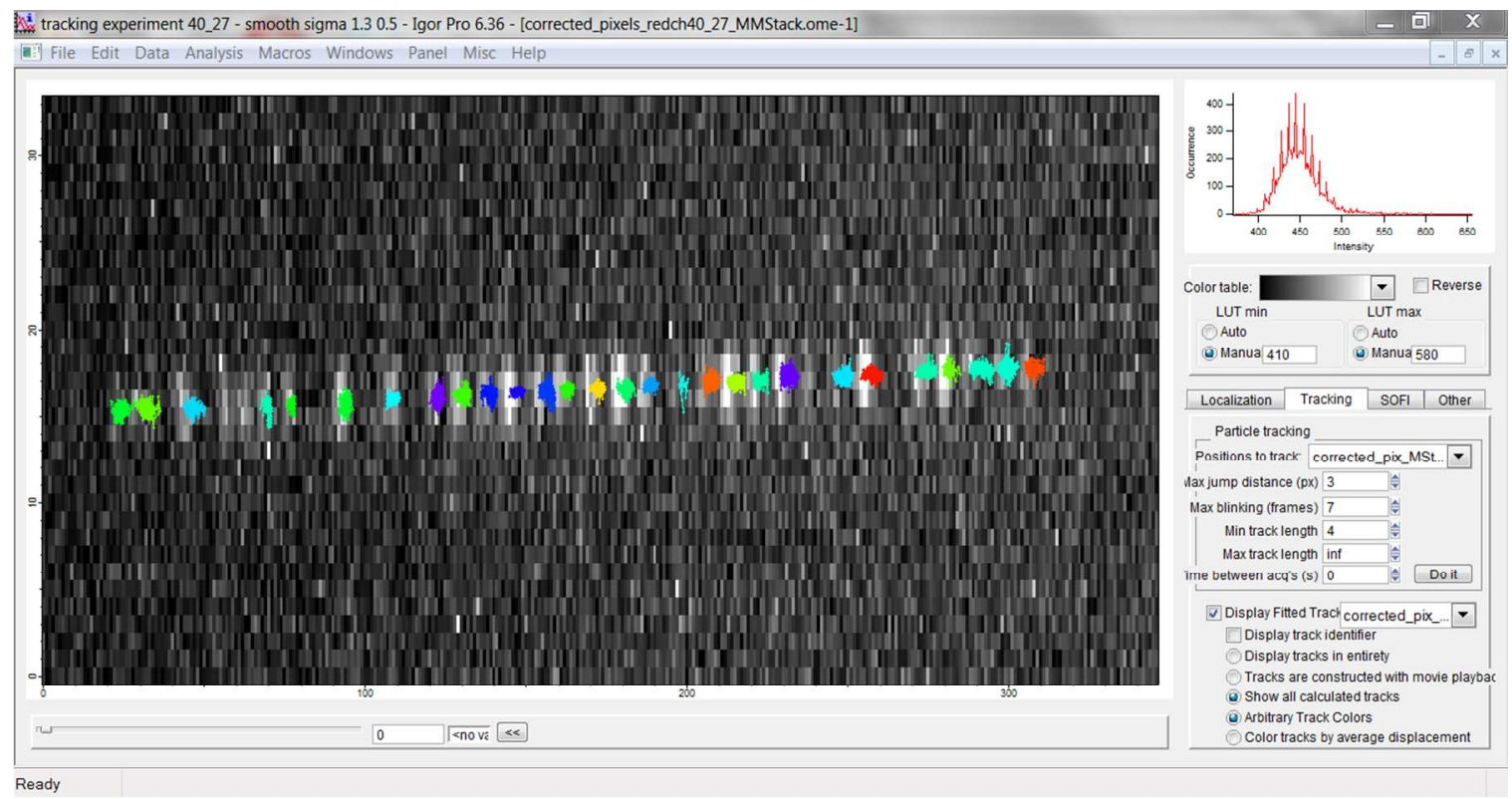

Figure S1. Localization and tracking with IgorPro Localizer plugin. The colored traces are the tracks of each fluorescent spot.

Distance calculation and histogram analysis: All tracks created with the IgorPro Localizer plugin were exported to a home-built Matlab R2015b (Mathworks ${ }^{\circledR}$ ) program to calculate the distances between the fluorescent labels. In each frame, the pairwise distances between the locations of all labels along a DNA repeat segment were calculated. For each molecule, the distances between labels belonging to the repetitive part of the DNA BAC molecule were plotted in a histogram with a bin size of 0.32 pixels. As can be seen in figure $\mathrm{S} 2$, these histograms show variations in the distributions between the molecules. While the standard deviation of the peaks remains relatively constant comparing different molecules, the distances between peaks in the distributions vary between them. This variation between molecules prohibits the use of a combined global distribution, since the underlying parameters of the distributions vary between molecules. Therefore, all the following analyses were molecule specific. 


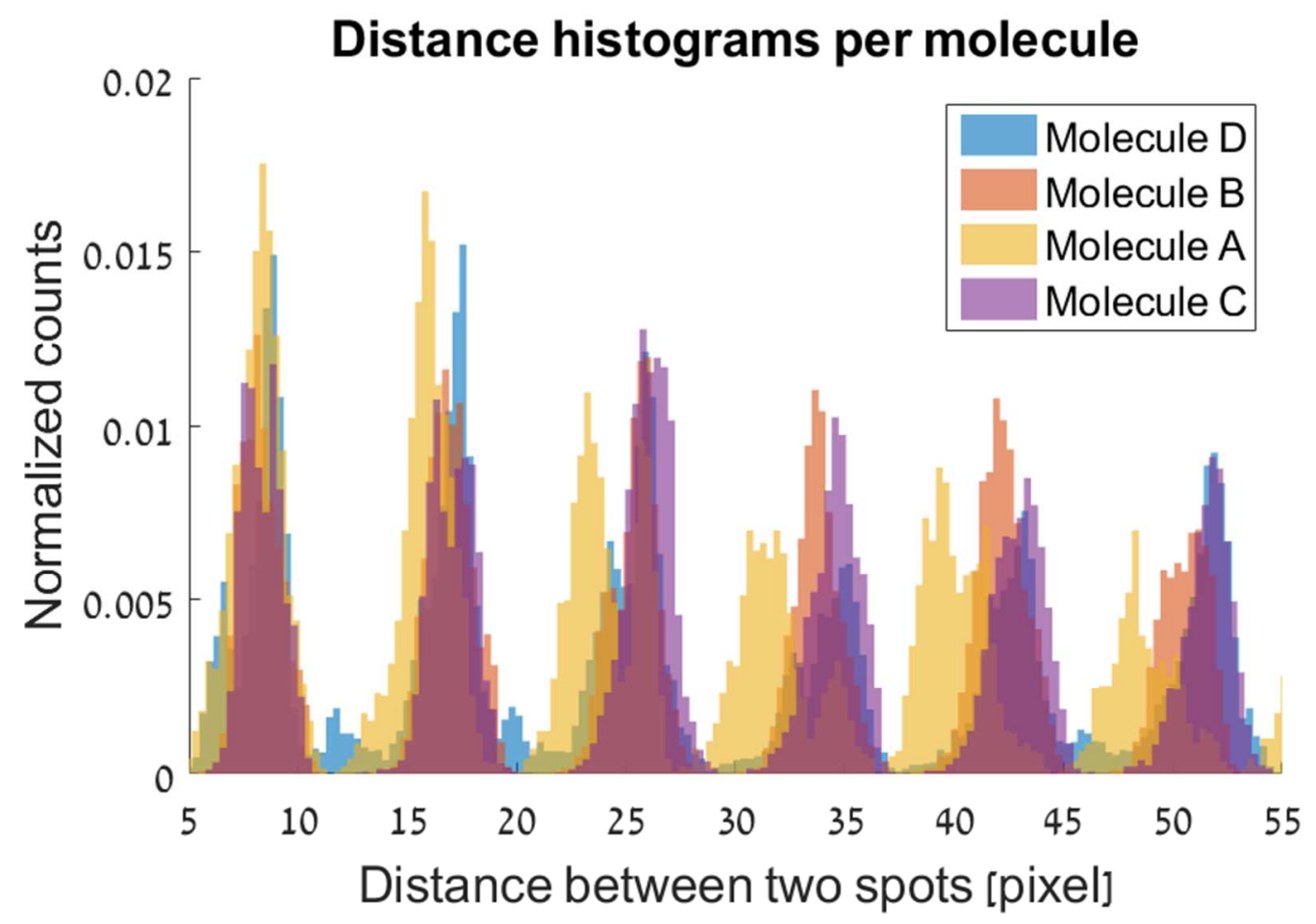

Figure S2. Distance distributions of the repetitive part of four different BAC molecules. The molecules are labeled according to the same annotation used in figures S3 and S4.

Gaussian fits to distance distributions: Since the first peak in the histogram represents the distances of labels separated by a single repetition segment, a Gaussian fit to this peak (see figure S3) yields the mean distance of these labels and its standard error (STE - standard deviation divided by the root number of measurements). The mean distance for this segment, found by using a first peak Gaussian fit (noted as FP), is: $\langle x\rangle_{F P-B}=1060.2 \pm 1.9 \mathrm{~nm},\langle x\rangle_{F P-C}=$ $1078.6 \pm 2.5 \mathrm{~nm}$ and $\langle x\rangle_{F P-A}=1074.3 \pm 3.2 \mathrm{~nm}$ (where the letters correspond to the numbering of the different $\mathrm{BAC}$ molecules presented in figure S3). Because of blinking and bleaching of the fluorescent labels, there is additional data corresponding to labels separated by more than the distance of a single repetitive element. By aggregating information from all pairwise distances, sampling may be increased and, therefore, another possibility for measuring the repetitive segment's length is fitting all distance distribution peaks to Gaussians and 
evaluating the mean distance by averaging over the differences between the mean distances of consecutive Gaussian peaks. The mean distance error in this method is calculated by averaging over the variances of the Gaussian peak errors and taking the standard error of that result. Fitting all the peaks to Gaussians (noted as AP), the mean distance of this segment is:

$\langle x\rangle_{A P-B}=1101.5 \pm 1.3 \mathrm{~nm}, \quad\langle x\rangle_{A P-C}=1118.9 \pm 1.6 \mathrm{~nm}$ and $\langle x\rangle_{A P-A}=1072.3 \pm$ $2.8 \mathrm{~nm}$
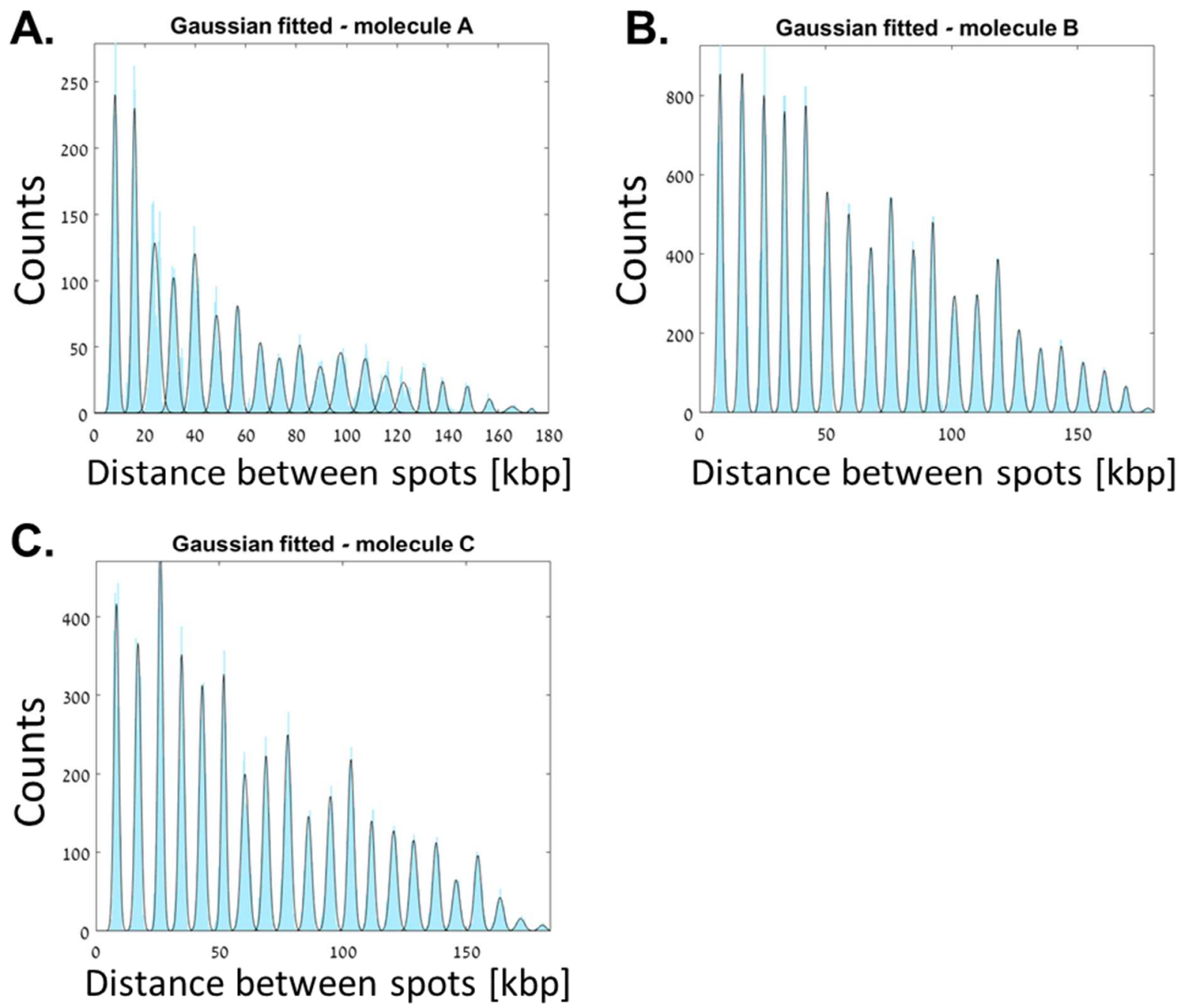
Figure S3. Distance distributions of different repetitive BAC molecules with single Gaussians fitted to the peaks of the distributions.

Odijk's theory and its fit to distance distribution: Odijk's regime describes the behavior of polymers confined to geometries with dimensions smaller than the polymer's persistence length. On the basis of free energy considerations, Su et al. showed that in this regime the probability distribution describing the likelihood of finding two points, separated by a contour length L, at a distance $x$ from one another is given $b^{2}$ :

$$
\text { (1) } P(x)=P_{0} \exp \left(B x-\frac{A}{L-x}\right)
$$

where $P_{0}$ is a normalization factor, $A=\frac{L^{2}}{4 \xi_{p}}, B=\frac{4 c^{2} \xi_{p}^{\frac{1}{3}}}{D^{\frac{4}{3}}}$ are two constants with $L$ being the contour length, $\xi_{p}=58 \mathrm{~nm}$ being the persistence length ${ }^{3}$, and $\mathrm{D}=45 \mathrm{~nm}$ being the channel width. In this analysis we set the constant $\mathrm{c}$, which appears in previous works ${ }^{2,4}$ but has no physical interpretation, to unity, so that the only free parameter in our experimental model is the average base pair size, which can vary due to YOYO labeling density ${ }^{3,5}$. From this equation one can calculate the most probable value of $x\left(X_{\max }\right)$ :

$$
\text { (2) } \frac{\partial P(x)}{\partial x}=0 ; P(x)=P_{0} \exp \left(B x-\frac{A}{L-x}\right) \quad \Rightarrow \quad X_{\max }=L-\sqrt{\frac{A}{B}}=L\left(1-\frac{1}{4 c}\left(\frac{\mathrm{D}}{\xi_{\mathrm{p}}}\right)^{\frac{2}{3}}\right)
$$

This result is exactly the same as that found using a different approach in the work of Su et $a l^{2}$. Furthermore, they showed that the standard deviation as a function of $X_{\max }$ can be calculated to be: 
(3) $\sigma_{\text {Odijk }}=\frac{D}{2 \sqrt{8 c^{3} \xi_{p}}} \sqrt{L}=\frac{D}{2 \sqrt{8 c^{3} \xi_{p}\left(1-\frac{1}{4 c}\left(\frac{D}{\xi_{p}}\right)^{\frac{2}{3}}\right)}} \sqrt{X_{\max }}$

When fitting equation 1 to measured localized data, as in our case (figure S3), one has to take into account the localization errors of the distances. As stated above, the 2D Gaussian localization method introduces an error that is dependent on the point spread function (PSF) of the localized fluorescent label and the amount of photons detected in that frame. Therefore, the actual distance probability distribution would be a convolution of Odijk's theoretic distribution (equation 1) and a Gaussian distribution with a standard deviation corresponding to the mean localization error:

(4) $P(x)=P_{\text {odijk }} * P_{\text {localization err }}=P_{0} \int_{-\infty}^{\infty} \exp \left(B \tau-\frac{A}{L-\tau}\right) \cdot \exp \left(-\frac{(x-\tau)^{2}}{2 \sigma_{\text {localization }}^{2}}\right) d \tau$

where $\sigma_{\text {localization }} \approx 0.3$ pixels is the mean localization error in our experiment, and $\tau$ is a summing variable. With this localization error, the total standard deviation of the mean distance between labels is expected to be larger due to the summed contribution of both distributions $\left(\sigma_{\text {tot }}=\sqrt{\sigma_{\text {Odijk }}^{2}+\sigma_{\text {localization }}^{2}}\right)$. Even with this implementation, the standard deviation of the measured distribution is higher than the theoretical expectation, as discussed in the main text.

As the width of the confining channels is well defined and consistent along the array ${ }^{6}$, and the persistence length of DNA in the used buffer is well known ${ }^{3}$, the average bp size conversion to $\mathrm{nm}$ (which effects the contour length) is the only physical variable available that can be used for fitting the Odijk distribution to the data. As was already established ${ }^{3,5,7}$, the average size of a bp can be approximated to be linearly dependent on the labeling density of the intercalating dye YOYO: the higher the density (up to a saturation of 1:4 dye:bp) the larger the effective bp size. 
As seen in the individual distance distributions (figure S4), each of the DNA molecules was uniformly stained with YOYO, resulting in a homogenous label distribution in the repetitive segment of the BAC. Therefore, the best fit to the Odijk distribution enables determining the average bp size with good accuracy and, thus, determining the YOYO labeling density of individual molecules. In figure S4 numerous fits of equation 1 were performed for each peak of the distance distributions, while keeping all the parameters set and changing the contour length by integer multiples of $3300 \mathrm{bp}$. The only parameter varied between different molecule distributions was the bp size conversion to $\mathrm{nm}$ (this was set for a single distribution for all the peaks).

A.

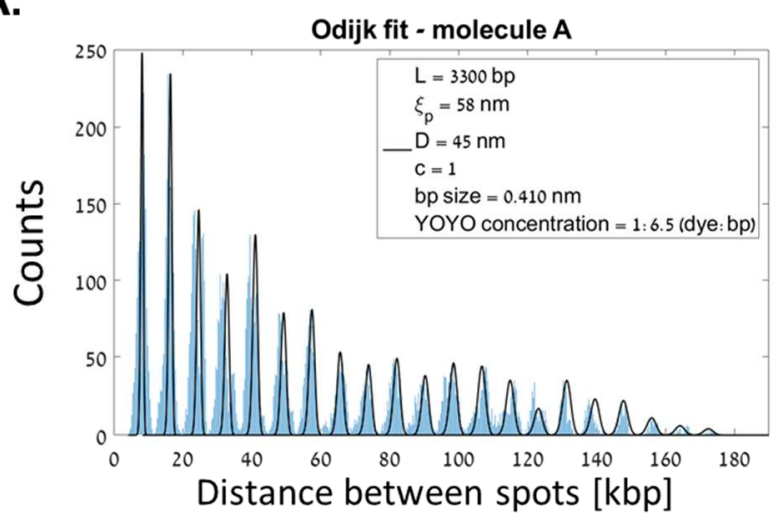

C.

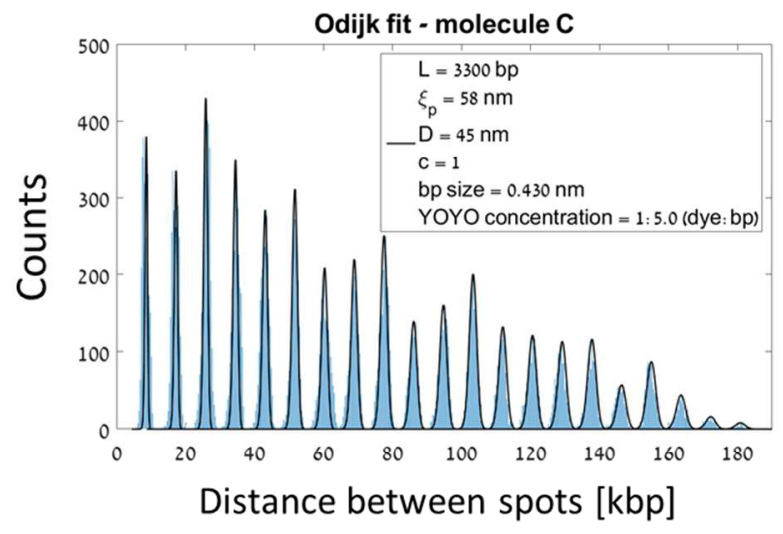

B.

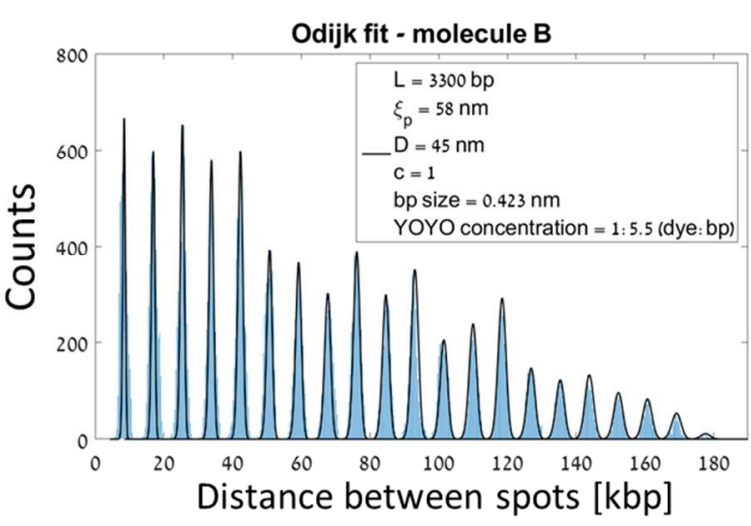

D.

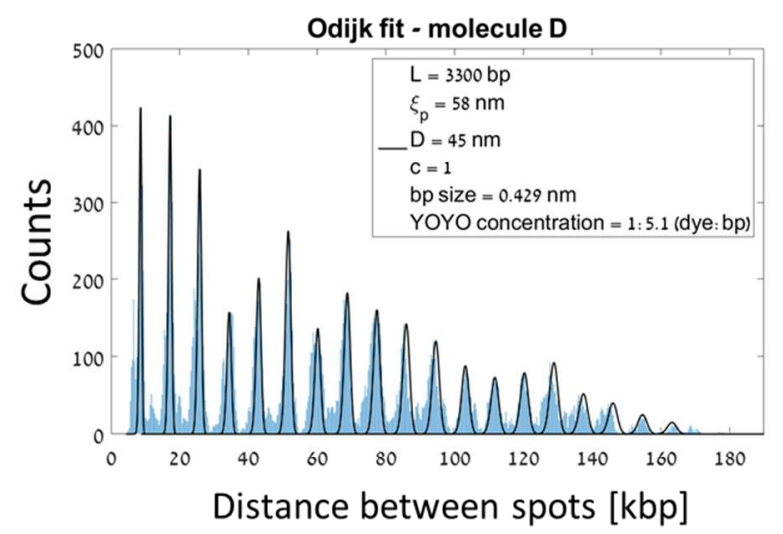


Figure S4. Odijk distribution fitted to distance distributions of four different molecules. The fit parameters are displayed in the boxes. The only parameter used for fitting was the average bp size (from which the YOYO labeling density was calculated as explained in the text).

Using the measurements reported by Gupta et al. ${ }^{3}$ that indicate that the contour length of a fully loaded $\boldsymbol{\lambda}$-DNA (1:4 YOYO:bp) is $22 \mu \mathrm{m}$ (comprising $48.5 \mathrm{kbp}$ ), while an unloaded $\boldsymbol{\lambda}$-DNA contour length is $16.5 \mu \mathrm{m}$, we linearized the base extension factor to calculate the concentration of YOYO staining in our own data. Using the average bp size from the Odijk distribution fit and the ratio calculated from the $\lambda$-DNA measurements, the single molecule's YOYO labeling density ranged from 1:5 to 1:6.5 dye per bp (assuming that is the only variable that affects the contour length and the difference between the molecules). The dependence of the contour length on the amount of intercalated YOYO dictates another constraint regarding the accuracy of distances between marks. This is because the bp size dependence on the YOYO labeling density affects the most probable distance between spots $\left(X_{\max }\right)$. The calculated value of $\mathrm{L}$ is $1419 \mathrm{~nm}$ for a 1:5.0 dye/bp ratio and $1353 \mathrm{~nm}$ when a 1:6.5 dye/bp ratio is used (theoretical $\mathrm{L}$ is $3.3 \mathrm{kbp}$ for both cases). Therefore, following equation $2, X_{\max }$ was calculated to be $1119 \mathrm{~nm}$ when a 1:5.0 dye/bp ratio was used and $1067 \mathrm{~nm}$ when a 1:6.5 dye/bp ratio was used. The variation between different molecules (up to $70 \mathrm{~nm}$ difference in the contour length and up to $52 \mathrm{~nm}$ difference between the imaged distances) is apparent when trying to estimate the distance between two close spots in the repetition array. Furthermore, to validate that the inconsistency originated from YOYO labeling density variation and was not due to inconsistency in channel widths, a comparison of the two parameters was made to evaluate their relative effect on the variance in distributions. We analyzed $X_{\max }$ according to equation 2 for different channel widths and bp sizes and found that in order to accommodate the differences in the measured distances between 
close labels one would have to introduce a variance of up to $13 \mathrm{~nm}$ in channel widths (see figure S5), which is unlikely. To conclude, these differences in the effective extensions of the molecules are additive to and are independent of all other inaccuracies, due to the induced shift of the entire distance distribution that they manifest. This inaccuracy can be mitigated by scaling each mapped molecule using reference points along the molecule and by a longer incubation of YOYO to increase YOYO staining homogeneity between different DNA molecules as shown by Nyberg et al. ${ }^{8}$.

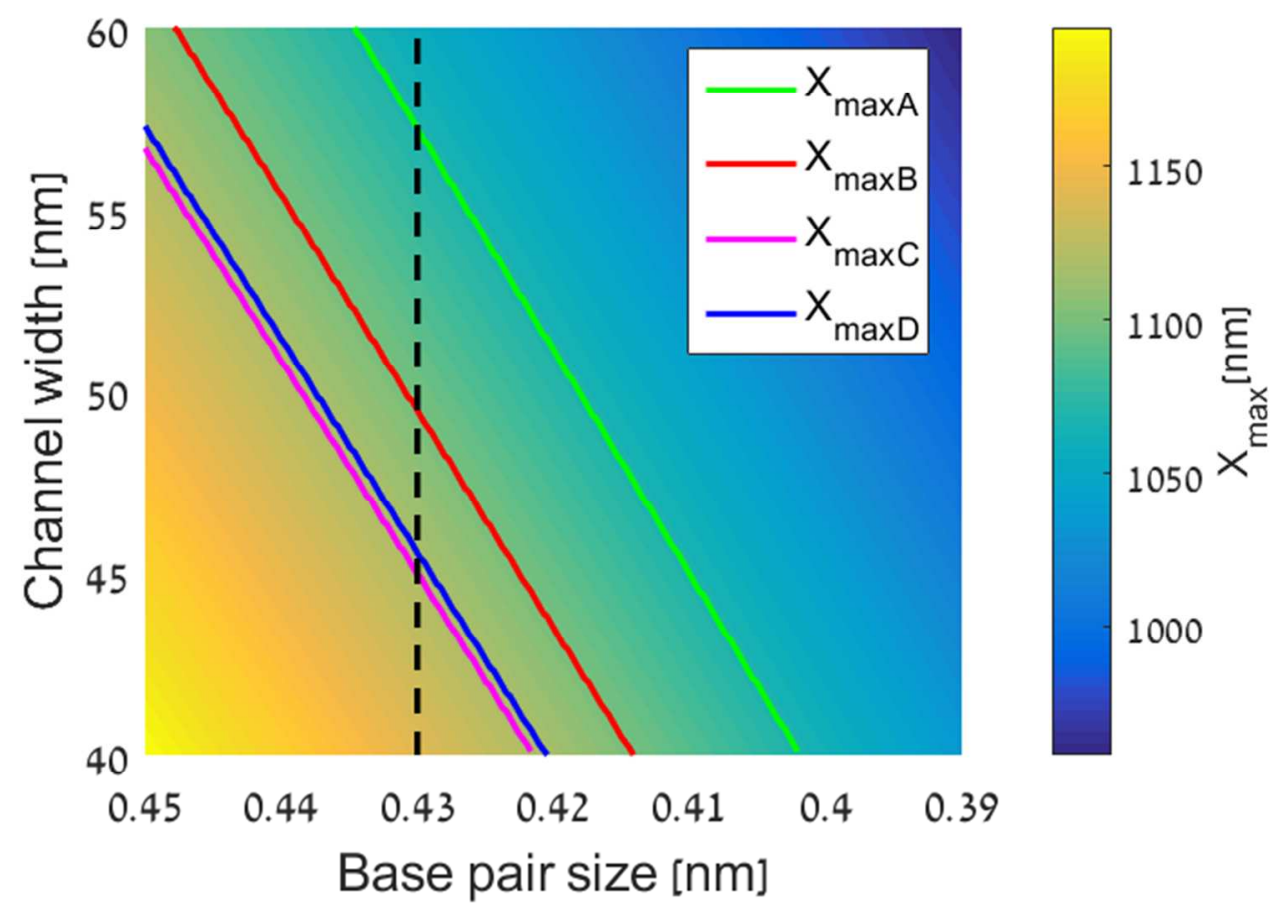

Figure S5. $X_{\max }$ plot as a function of bp size and channel width, the color bar represents $X_{\max }$ values and the colored lines show the calculated $X_{\max }$ extensions for the molecules in figure S4. Following the dashed line in the plot (representing a constant bp size) it is evident that a change of $13 \mathrm{~nm}$ in channel width is needed to explain the variation in $X_{\max }$. Therefore, the YOYO labeling density would be a more suitable variable for fitting the distance distribution to theory. 
Stretching factor and $\mathbf{n m}$ to bp conversion calculation: In order to be able to convert between image plane distances calculated in $\mathrm{nm}$ from the acquired images and the actual genomic distances, a conversion factor needs to be calculated. For this purpose we employed three different methods (as explained above) to extract the mean distance between adjacent repetitive labels on the BAC DNA (here we present the values for one of the BAC molecules analyzed): a Gaussian fit to a single peak in the distance distribution $\left(\langle x\rangle_{F P-B}=1060.2 \pm\right.$ $1.9 \mathrm{~nm})$, Gaussians fits to all peaks in the distance distribution $\left(<x>_{A P-B}=1101.5 \pm 1.3 \mathrm{~nm}\right)$ and finally Odijk's distribution fit to the distance distribution data and calculation of the most probable distance according to the fit parameters $\left(X_{\max -B}=1100 \mathrm{~nm}\right)$. As can be seen above, all methods give roughly the same estimation, with the latter two more accurately describing the entire distance distribution and, hence, probably giving a better estimation.

Mapping accuracy comparison - alignment analysis: In order to estimate the contribution of time averaging to the genome mapping accuracy, we compared the alignment scores of the same BAC molecule (molecule B) with and without time averaging. Single frames from the time lapse were aligned to the theoretical genome, and their alignment scores were compared to the scores of the time averaged label locations. The calculation of the time averaged label locations was performed, as explained in the main text, by two different methods, and the results are presented in figure 4. The alignment was conducted using two genome mapping alignment programs: the commercial IrysView program (by BioNano Genomics Inc. ${ }^{6}$ ) and a cross-correlation (CC) kymograph based alignment algorithm ${ }^{9,10}$.

The CC alignment software was designed to align an intensity profile of labeled DNA with a simulated profile derived from the known sequence. The output score of this analysis, as explained previously ${ }^{10}$, is the numerical CC value defining the degree of similarity between the 
tested and theoretical profiles. In order to comply with the input requirements a custom Matlab program converted the label locations to a simulated intensity image by plotting Gaussians centered at the labels' locations with standard deviations of $\sim 130 \mathrm{~nm}$, as indicated by the measurements. Alignment was conducted on the spot patterns extracted from the two frames with the maximal number of detected labels (frames 14 and 31 - 19 detected labels from the repetitive segment of the BAC molecule), as well as for the averaged label patterns calculated by the two averaging methods. Since CC alignment is very sensitive to missing data (due to blinking/bleaching of the fluorescent labels) the averaged data was based on 19 of the 20 labels that were initially detected, in order to perform an unbiased comparison with the single frame data. The four simulated images were aligned to the repetitive theoretical sequence of a BAC with 19 repeats, using the $\mathrm{CC}$ alignment software and their cross-correlation scores were compared (figure S7). In order to eliminate any bias to the scores originating from the simulation itself, a simulation of the theoretic sequence was also executed and aligned to the same sequence. All the scores were normalized according to this result. From this analysis it is clear (as also explained in the main text) that by averaging over multiple frames one can generate better alignment accuracy than can be achieved with a single frame location, due to the recovery of the equilibrium positions of the labels.

In order to estimate the number of frames required for an improvement in alignment score, cumulative average distances were calculated for an increasing number of frames. For each set of frames, an intensity profile was simulated according to the averaged locations and aligned to a 20 labels repetitive sequence reference (figure S7B). As seen both in the IrysView analysis shown in the main text (figure 5B) and in the CC alignment analysis (figure S7B), a significant increase in score is evident after $\sim 50$ averaged frames. 


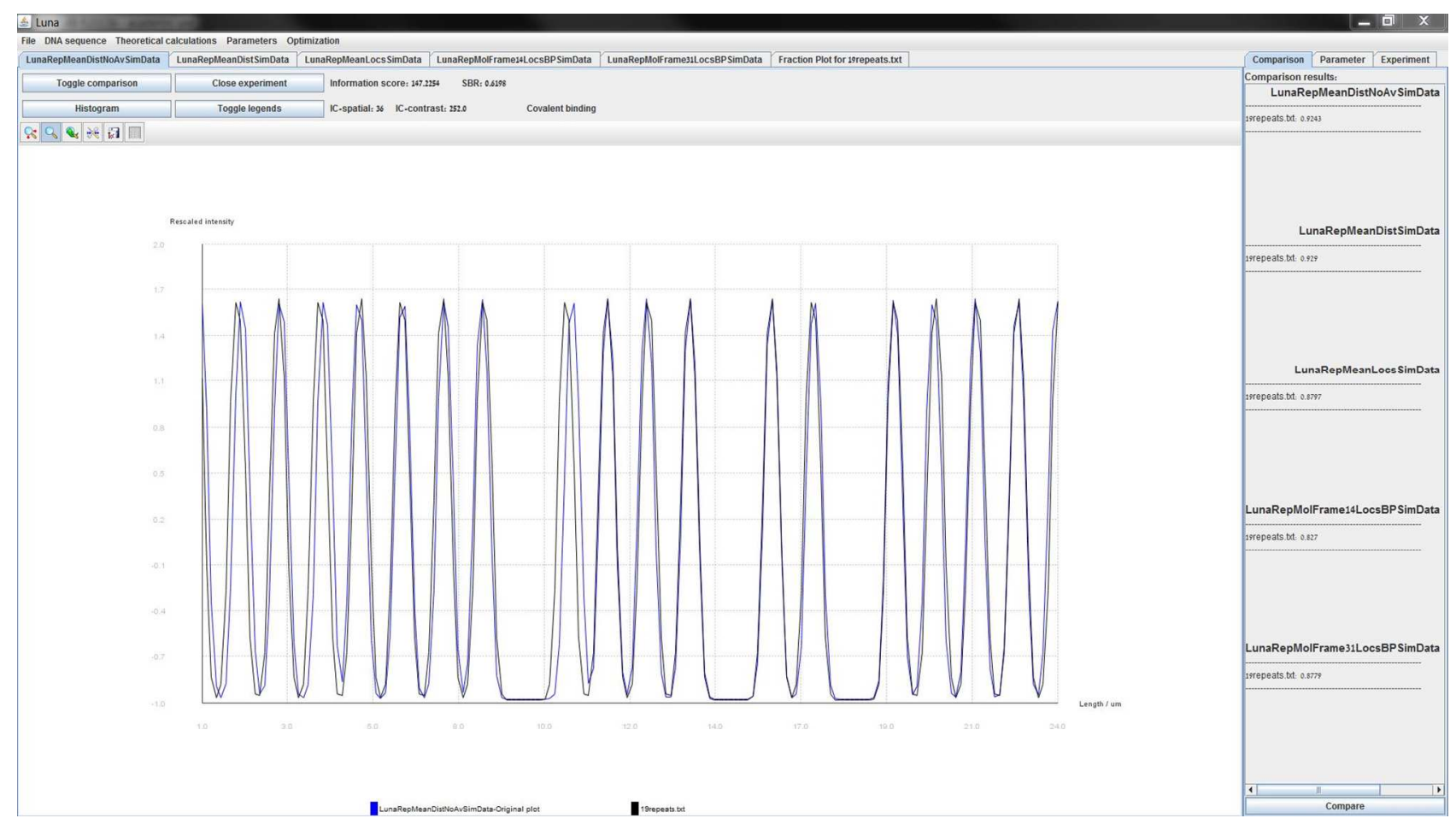

Figure S6. An example of the CC alignment software trace. We aligned two types of averaged label profiles and two single frames to a 19 label theoretical reference. 
A.

CC scores - cumulative average
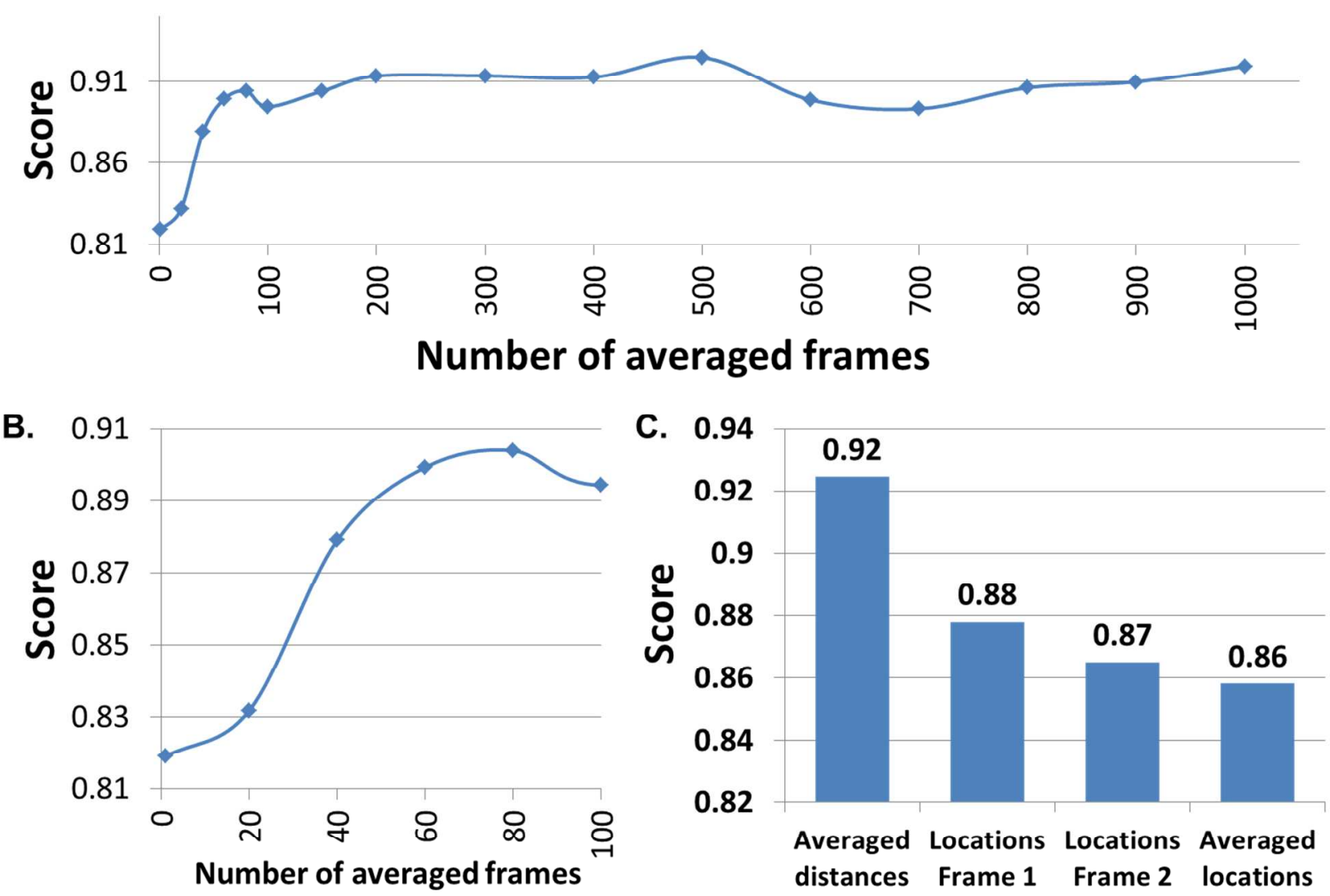

Figure S7. Alignment score comparison using the $\mathrm{CC}$ alignment software. (A) Alignment score as a function of number of averaged frames. (B) Zoom-in on the first 100 frames of graph A. The averaged distances for alignment were calculated in incremental steps of 20 frames. (C) Alignment score comparison between the cumulative averaged distances, single frame snapshots and the averaged locations calculation. All locations were fitted to a 19 labels theoretical sequence.

In order to compare alignment scores calculated by the commercial IrysView program we generated genetic maps based on the label locations calculated for the averaged, as well as for the individual, frames. These were aligned to a reference map calculated for the entire BAC 
sequence (consisting of 21 repeats). As mentioned before, since the amount of labels emitting light varied between frames due to bleaching and blinking, we deliberately omitted extra labels in the confidence score calculations presented in the main text in order to generate an unbiased comparison. In our experiments, only the averaged data contained representation of all genetic labels; the maximum number of labels simultaneously detected in a single frame was 27 while a total of 28 labels were captured by frame averaging. This extra information provided by multiframe analysis contributes to an additional increase in alignment score. A comparison considering all labels is presented in figure $\mathrm{S} 8$ and shows better scores compared to the single frame snapshots. This emphasizes yet another advantage of the averaging approach, which is the increased ability to overcome bleaching or blinking events in mapping a molecule to the reference genome. The alignment scores are presented in the main text in figure 5. The confidence score given by the IrysView program for a certain alignment corresponds to $-\log \left(P_{\text {value }}\right)$, where $P_{\text {value }}$ is the probability that the molecule is unrelated to the compared sequence and the alignment was purely by chance. ${ }^{11}$ Therefore, a one point increase in score means an increase of one order of magnitude in certainty of alignment. 
A.
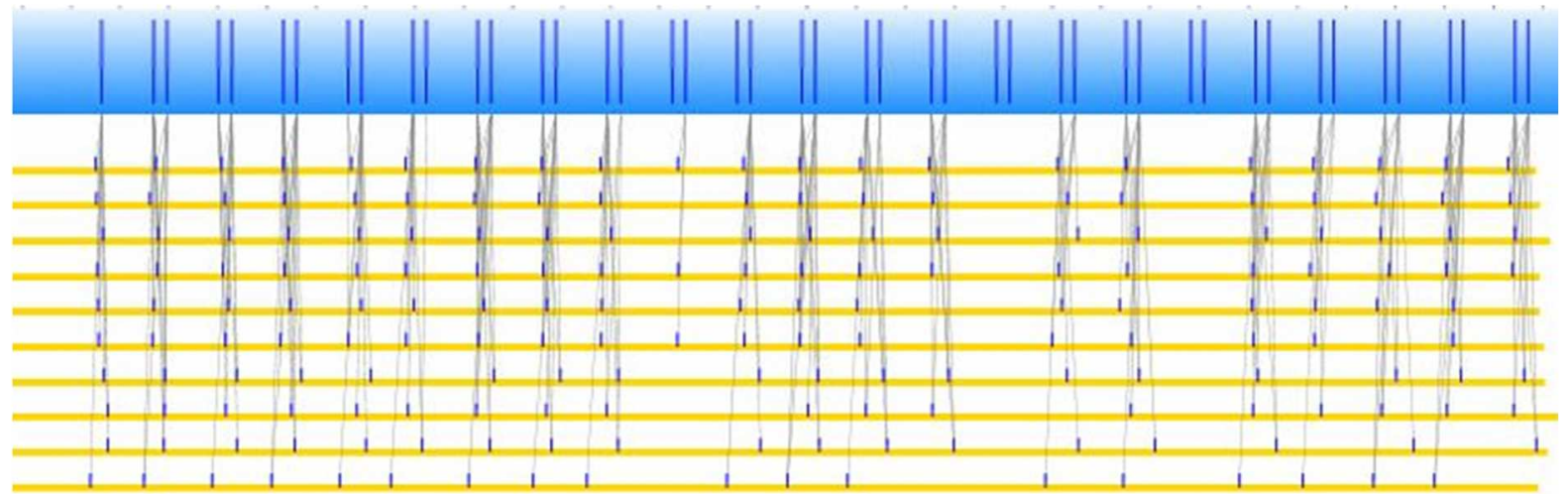

B.

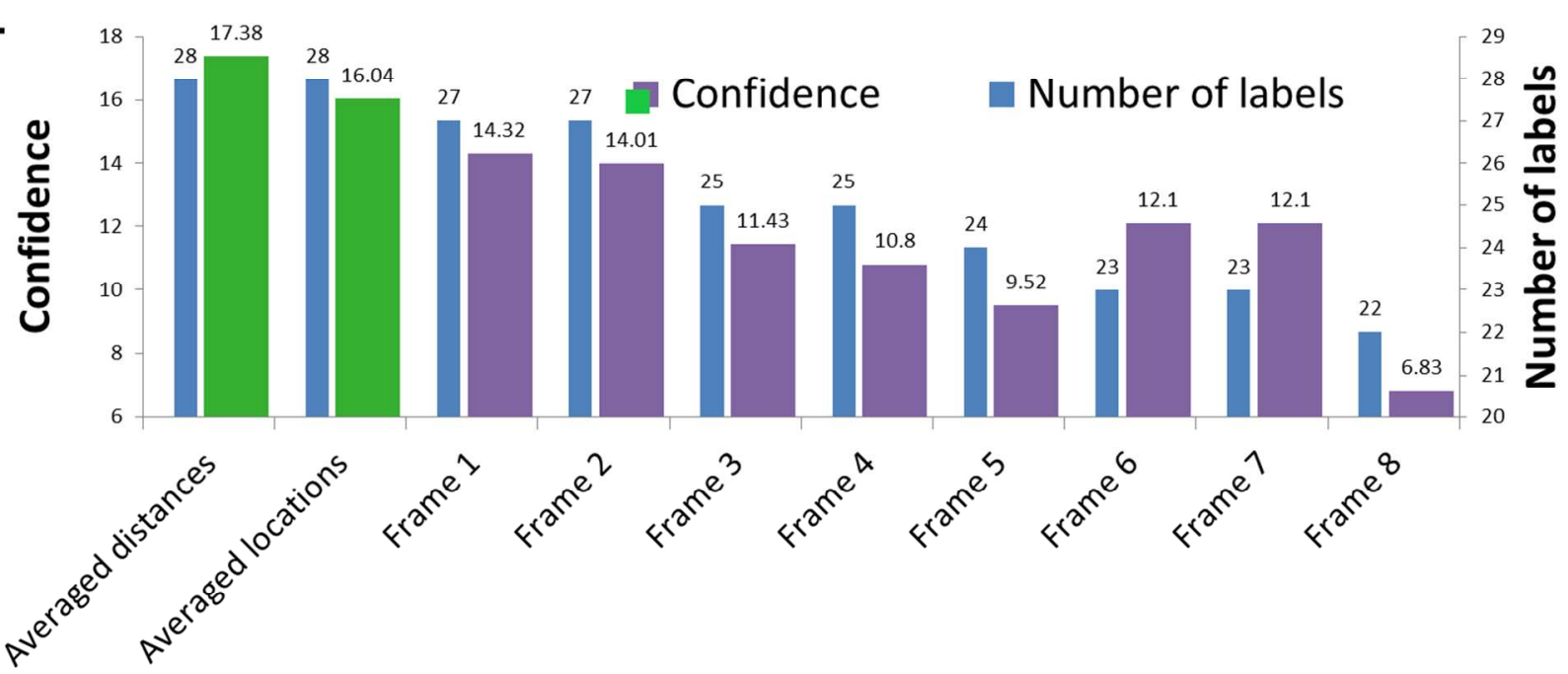

Figure S8. IrysView alignment comparison without omitting labels. (A) Graphic representation of the molecules' alignment to the theoretical sequence generated by IrysView. Molecules are represented as yellow lines with labels marked as blue bars. The theoretical sequence is presented on top, with TCGA expected labeling marked as blue lines across the sequence. (B) Alignment score comparison between the averaged label locations and single frame label locations. The graph shows the alignment scores next to the amount of labels that were aligned (this is not constant due to bleaching and blinking effects).

Super-resolution analysis: The distances between the reference label and the target label for super-resolution were calculated in each frame according to the localization data acquired with the Localizer software ${ }^{1}$. The bleaching frame of the target label was determined by the sum of 
the pixel intensities of each localized label. The mean distance errors were defined as the standard deviation of the distances along the averaged frames.

A.

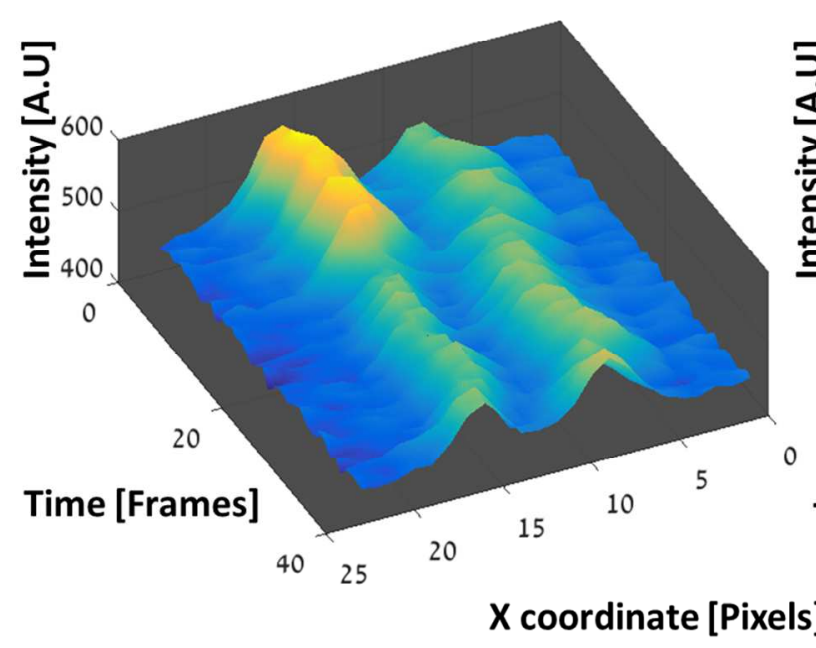

B.

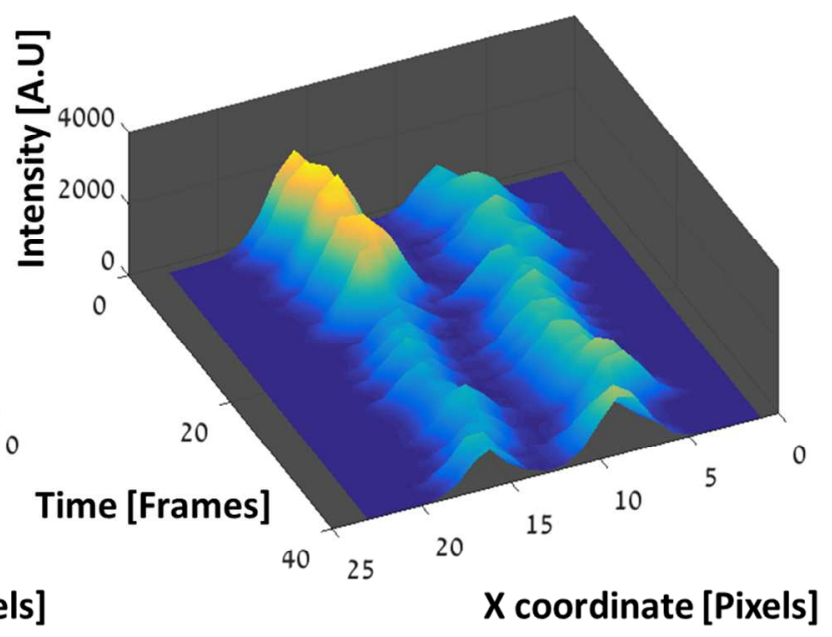

Figure S9. Comparison of the raw intensity kymograph (A) versus the kymograph simulated according to the localization data (B).
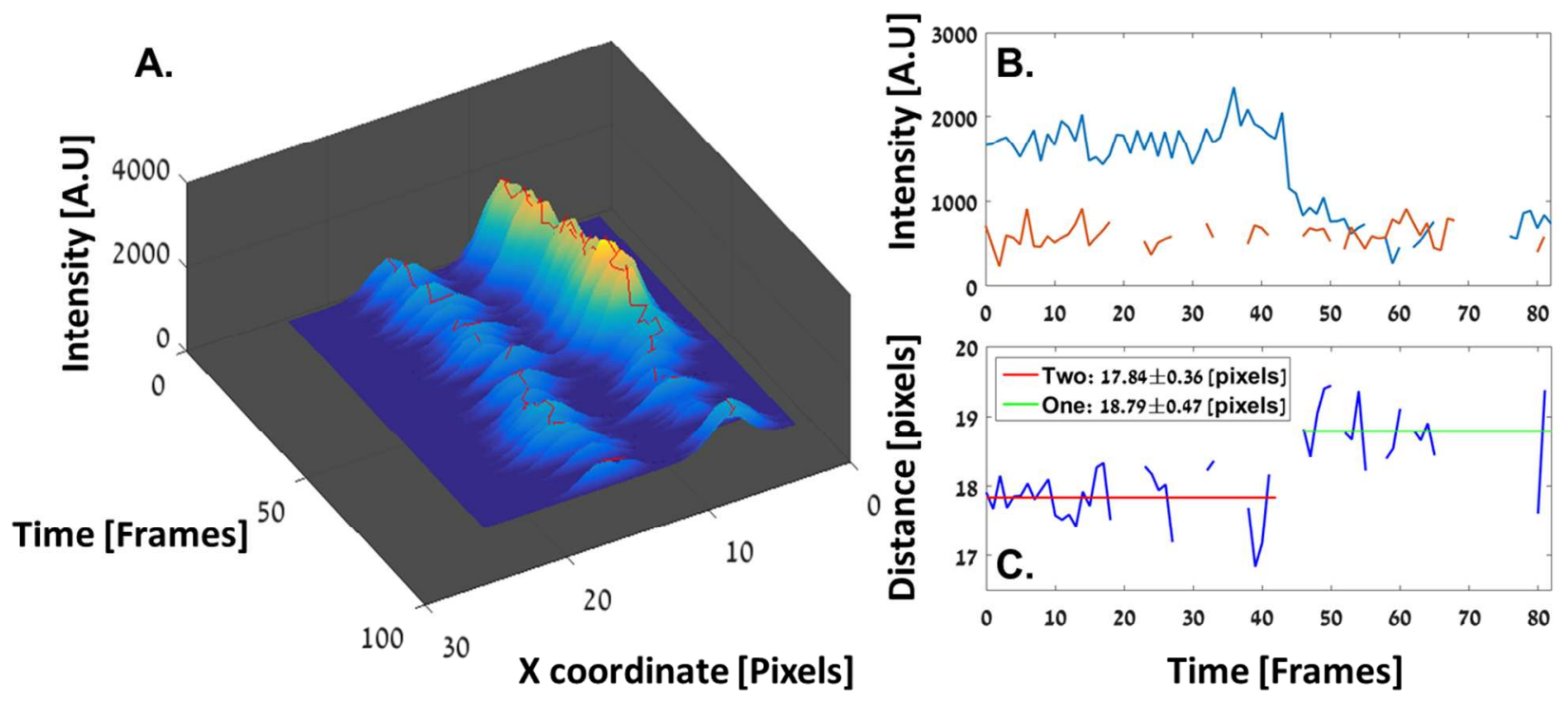
Figure S10. Super-resolution of reference and target labels separated by $6.6 \mathrm{kbp}$. The calculated distance between the sub-diffraction-spaced fluorophores comprising the target spot is $741 \pm 230$ bp (1.9 \pm 0.59 pixels). The gaps in graphs $\mathrm{B}$ and $\mathrm{C}$ arise from missing data due to SNR values that were too low for localization in these frames.

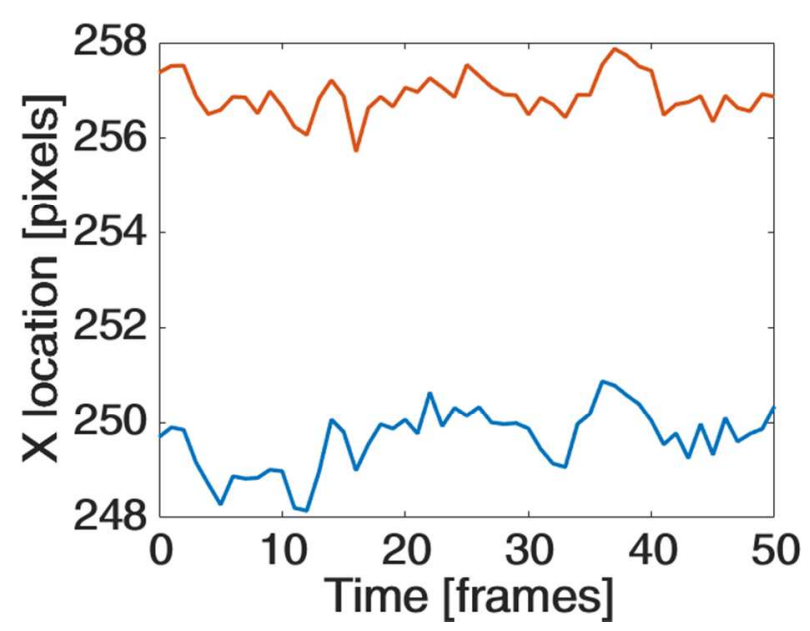

Figure S11. X location kymograph for the two spots presented in figure 6 (main text). The global fluctuations prohibit resolution of the sub-diffraction fluorophore structure, even when a bleaching event occurs (after $\sim 15$ frames as seen in figure $6 \mathrm{~B} \& \mathrm{C}$ ).

In order for the super-resolution analysis to succeed the following requirements had to be fulfilled:

- A clear single bleaching/ blinking step had to be distinguishable in the target label's intensity. A short time frame (usually on the order of $\sim 50-100$ frames) around the event is used in order to avoid the screening nature of the thermal fluctuations over the distance averages. Consequently, in the more general case of more than two subdiffraction distanced fluorophores, subsequent bleaching steps would have to be 
temporally resolved with a minimum of $\sim 50-100$ frames between bleaching/blinking events, in order to spatially resolve their locations.

- A reference point must be found with sufficient localization data during the target's bleaching/ blinking event, meaning a significant SNR of the reference point and no significant blinking/bleaching of the reference point during the target's blinking/bleaching event.

- Distance of the target label from the reference point should not exceed $10 \mathrm{kbp}$, so that correlation between their fluctuations is sufficiently significant (see figure S12) to allow global fluctuations elimination by distance averaging. As the distance between the target and reference labels increases, the super-resolution calculation's accuracy diminishes (as can be seen in figure S10 compared to figure 6 in the main text).

Correlation between label locations: The correlation between label locations was calculated using Pearson's correlation coefficient ${ }^{12}$ as a function of the distance between labels. For each BAC molecule correlation between pairs of labels from the repetitive segment $\left(\rho_{i}\right)$ were calculated with the estimated error $\left(\sigma_{i}\right)$ defined as $95 \%$ confidence of the coefficient per pair (calculated by Matlab's built-in algorithm). The mean correlation coefficient per distance between labels $\left(<\rho_{\text {dist }}>\right)$ and its error were calculated as a weighted average of all the pairs of labels corresponding to that distance ${ }^{13}$ :

$$
\text { (5) }<\rho_{\text {dist }}>=\frac{\sum\left(\rho_{i} / \sigma_{i}^{2}\right)}{\sum 1 / \sigma_{i}^{2}} \pm \frac{1}{\sqrt{\sum 1 / \sigma_{i}^{2}}}
$$

It is evident from the results (figure S12) that, generally, the labels' fluctuations are well correlated within $\sim 7 \mathrm{kbp}$ and in some cases up to more than $\sim 30 \mathrm{kbp}$. These results explain the 
accuracy enhancement achieved by averaging over closest neighbor distances compared to averaged locations analysis, which is due to global fluctuation reduction in the former method. The results also support the use of the super-resolution method suggested in this text and provide a good estimation of the maximal distance allowed between the target label and the reference point.
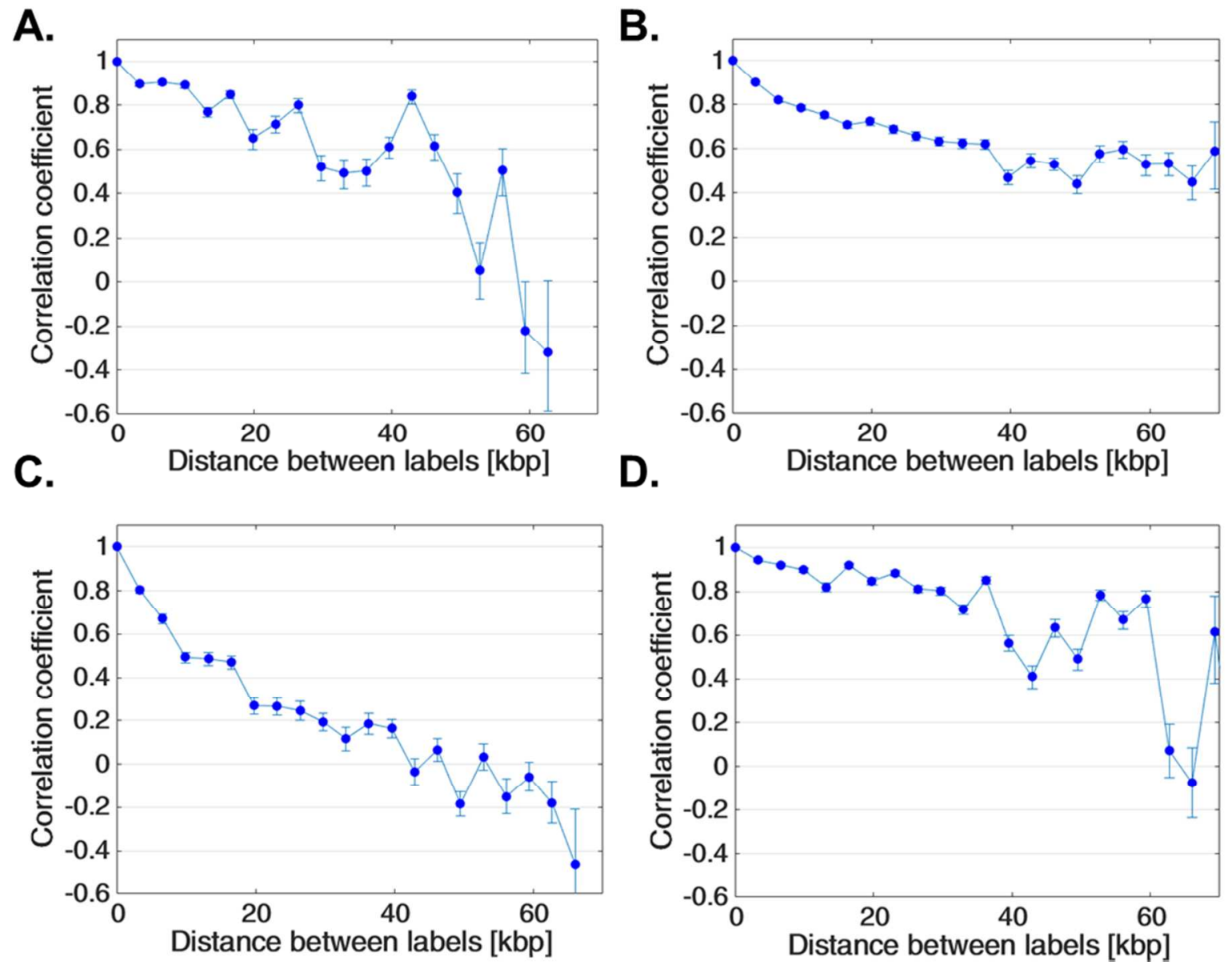

Figure S12. Pearson's correlation coefficient as a function of distance between labels. The molecules are labeled by the same letters as in figure S4

DBCO+Cy5+Trolox synthesis: All reactions requiring anhydrous conditions were performed in an Argon atmosphere. All reactions were carried out at room temperature, unless stated otherwise. Chemicals and solvents were either A.R. grade or purified by standard techniques. 
Thin layer chromatography (TLC): silica gel plates Merck 60 F254 and compounds were visualized by irradiation with UV light. Flash chromatography (FC): silica gel Merck 60 (particle size $0.040-0.063 \mathrm{~mm}$ ), eluent given in parentheses. High pressure liquid chromatography (HPLC): C18 5u, 250x4.6mm, eluent given in parentheses. Preparative HPLC: C18 5u, 250x $21 \mathrm{~mm}$, eluent given in parentheses. 1H-NMR spectra were measured using Bruker Avance operated at $400 \mathrm{MHz}$, as mentioned. All general reagents, including salts and solvents, were purchased from Sigma-Aldrich.

Chemical abbreviations: ACN - Acetonitrile, DCM - Dichloromethane, DMAP - 4Dimethylaminopyridine, DMF - N,N'-Dimethylformamide, EtOAc - Ethylacetate, Hex - nhexanes, MeOH - Methanol, THF - Tetrahydrofurane, TFA - Trifluoroacetic acid, Et3N Triethylamine, EtOH - Ethyl alcohol, NaOAc - Sodium acetate, Ac2O - Acetic anhydride, $\mathrm{AcOH}$ - Acetic acid, NaOAc - Sodium Acetate, NHS - N-Hydroxysuccinimide, DCC - N,N'Dicyclohexylcarbodiimide. 


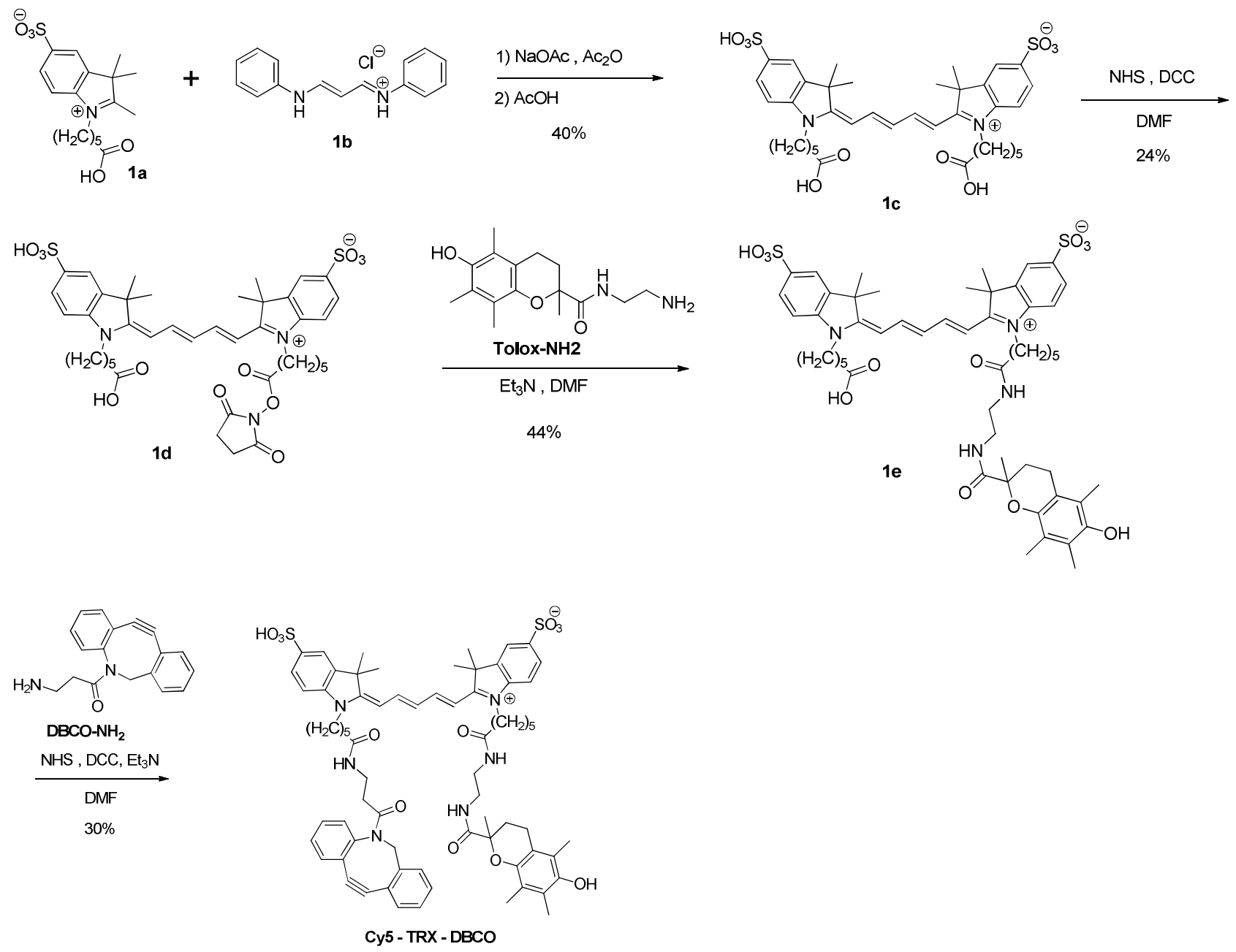

Figure S13. Synthesis of Cy5-Trolox-DBCO conjugate.

Compound $1 \mathrm{c}-$ Sulfonate indolium derivative 1a (100mg, 0.39mmol), NaOAc (140mg, $1.7 \mathrm{mmol})$ and 3-Anilinoacraldehyde anil hydrochloride $1 \mathrm{~b}(302 \mathrm{mg}, 0.85 \mathrm{mmol})$ were dissolved in $2 \mathrm{ml}$ of Ac2O. The reaction mixture was stirred for 1 hour at $80^{\circ} \mathrm{C}$ and was monitored by RPHPLC (grad. 10\%-90 ACN in water, 20min). After completion, the solvent was evaporated in vacuo. $\mathrm{Next}, 5 \mathrm{ml} \mathrm{AcOH}$ was added into the crude residue, followed by stirring for 1 hour at $50^{\circ} \mathrm{C}$. The solvent was concentrated by evaporation under reduced pressure. The crude product was diluted with 1:1 H2O:ACN, and purified by preparative RP-HPLC (grad. 10\%-90 ACN in water, $20 \mathrm{~min})$ to give product $1 \mathrm{c}(115 \mathrm{mg}, 40 \%)$. 
MS (ES+): m/z calc. for C37H46N2O10S2: 743.3; found: 742.3 [M-H]- , $743.3[\mathrm{M}]+$.
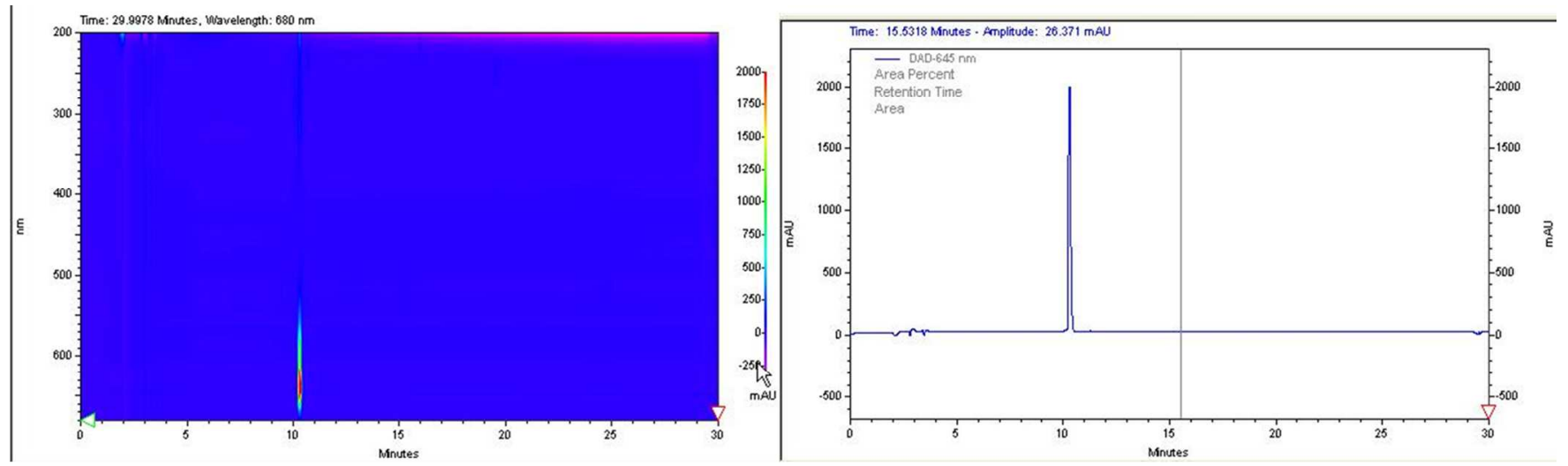

Figure S14. Compound 1c: Left: HPLC 3D chromatogram. Right: HPLC 2D chromatogram at $680 \mathrm{~nm}$.

Compound $1 \mathrm{~d}-1 \mathrm{c}(90 \mathrm{mg}, 0.12 \mathrm{mmol})$, DCC (75mg, 0.33mmol) and NHS (36mg, 0.33mmol) were added to a round-bottomed flask containing DMF. The reaction was stirred at room temperature and monitored by RP-HPLC (grad. 10\%-90 ACN in water, 20min). After completion, the reaction mixture was concentrated by evaporation under reduced pressure. The crude product was diluted with 1:1 $\mathrm{H} 2 \mathrm{O}: \mathrm{MeOH}$, and purified by preparative RP-HPLC (grad. $10 \%-90 \mathrm{ACN}$ in water, 20min) to give compound $1 \mathrm{~d}$ as a blue solid (24mg, $24 \%$ ).

MS (ES+): m/z calc. for C41H49N3O12S2: 840.3; found: 839.2 [M-H]- , 840.3 [M]+.
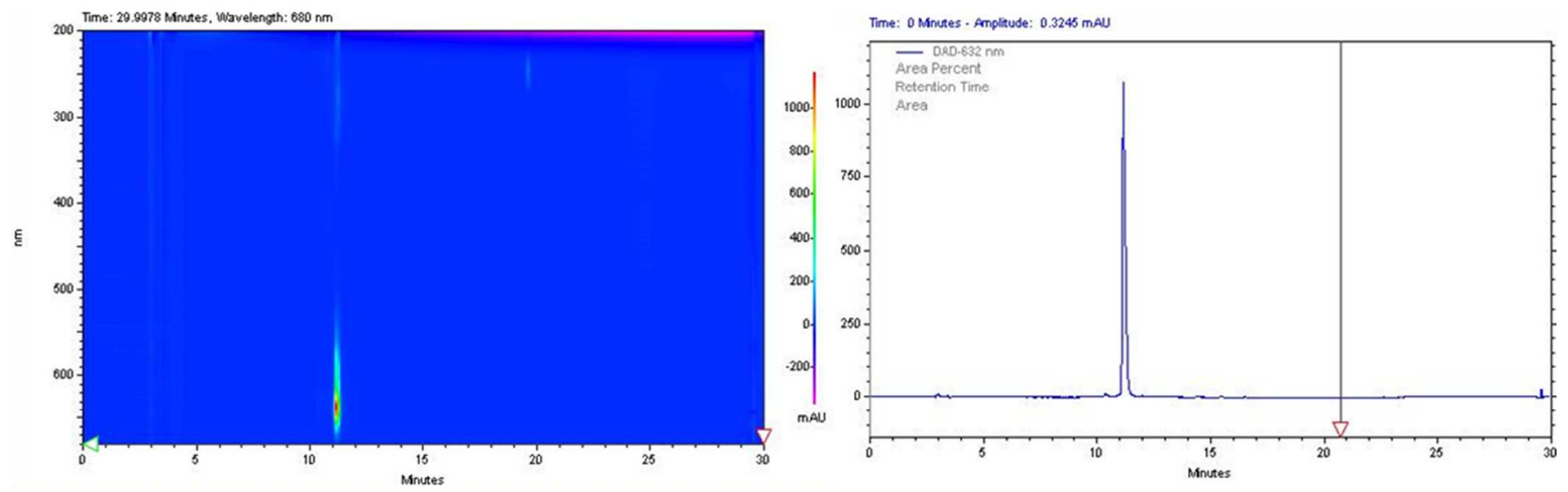
Figure S15. Compound 1d: Left: HPLC 3D chromatogram. Right: HPLC 2D chromatogram at $680 \mathrm{~nm}$.

Compound 1e - $1 \mathrm{ml}$ of DCM:TFA (1:1) was added to a round-bottomed flask containing Trolox-NH2 (6.3mg, $0.02 \mathrm{mmol})$. After $5 \mathrm{~min}$ of stirring, the solvent was evaporated in vacuo. Next, $1 \mathrm{ml}$ DMF was added, followed by the addition of $1 \mathrm{~d}(15 \mathrm{mg}, 0.02 \mathrm{mmol})$ and one drop of triethylamine. The reaction was stirred at room temperature and was monitored by RP-HPLC (grad. 10\%-90 ACN in water, 20min). After completion, the reaction mixture was concentrated by evaporation under reduced pressure. The crude product was diluted with 1:1 H2O:MeOH, and purified by preparative RP-HPLC (grad. 10\%-90 ACN in water, 20min) to give compound 1e as a blue solid (8mg, 44\%).

MS (ES+): m/z calc. for C53H68N4O12S2: 1017.4; found: 1016.4 [M-H]- , 1017.3 [M]+.
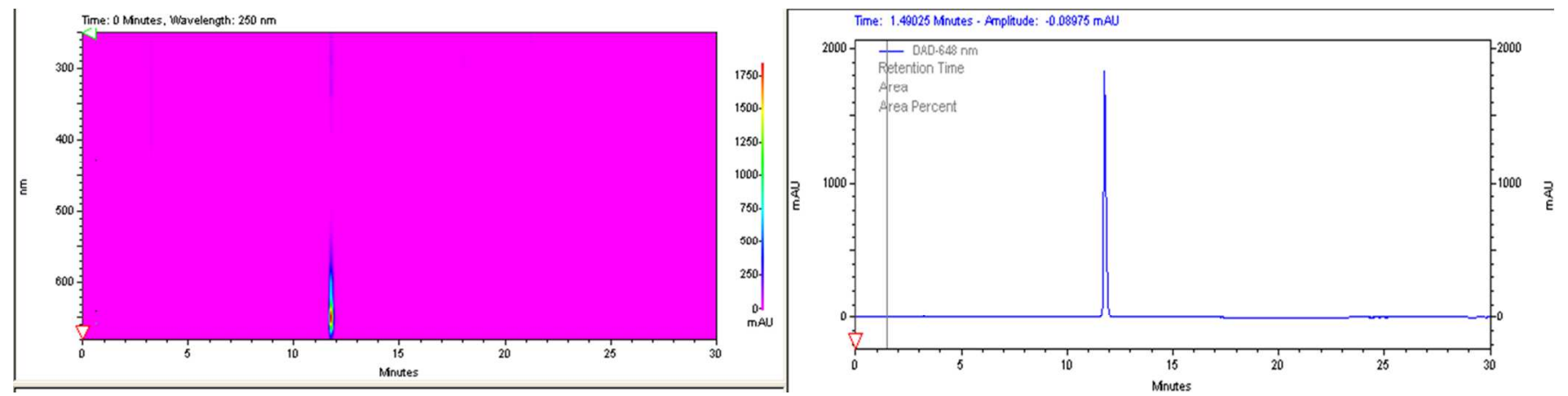

Figure S16. Compound 1e: Left: HPLC 3D chromatogram. Right: HPLC 2D chromatogram at $680 \mathrm{~nm}$.

Cy5-Trolox-DBCO (final compound) - Compound 1e (8mg, $8 \mu \mathrm{mol})$, DCC (3.25mg, 16 $\mu \mathrm{mol})$, NHS $(1.8 \mathrm{mg}, 16 \mu \mathrm{mol})$, one drop of triethylamine and commercially available DBCO-NH2 (3.25mg, $12 \mu \mathrm{mol})$ were added to a round-bottomed flask containing DMF. The reaction was heated to $50^{\circ} \mathrm{C}$ and was monitored by RP-HPLC (grad. 10\%-90 ACN in water, 20min). After 
completion, the reaction mixture was concentrated by evaporation under reduced pressure. The crude product was diluted with 1:1 $\mathrm{H} 2 \mathrm{O}: \mathrm{MeOH}$, and purified by preparative RP-HPLC (grad. 10\%-90 ACN in water, 20min) to give compound Cy5 - TRX - DBCO as a blue solid (3mg, $30 \%)$.

MS (ES+): m/z calc. for C71H82N6O12S2: 1274.5; found: 1273.3 [M-H]- , 1274.5 [M]+.
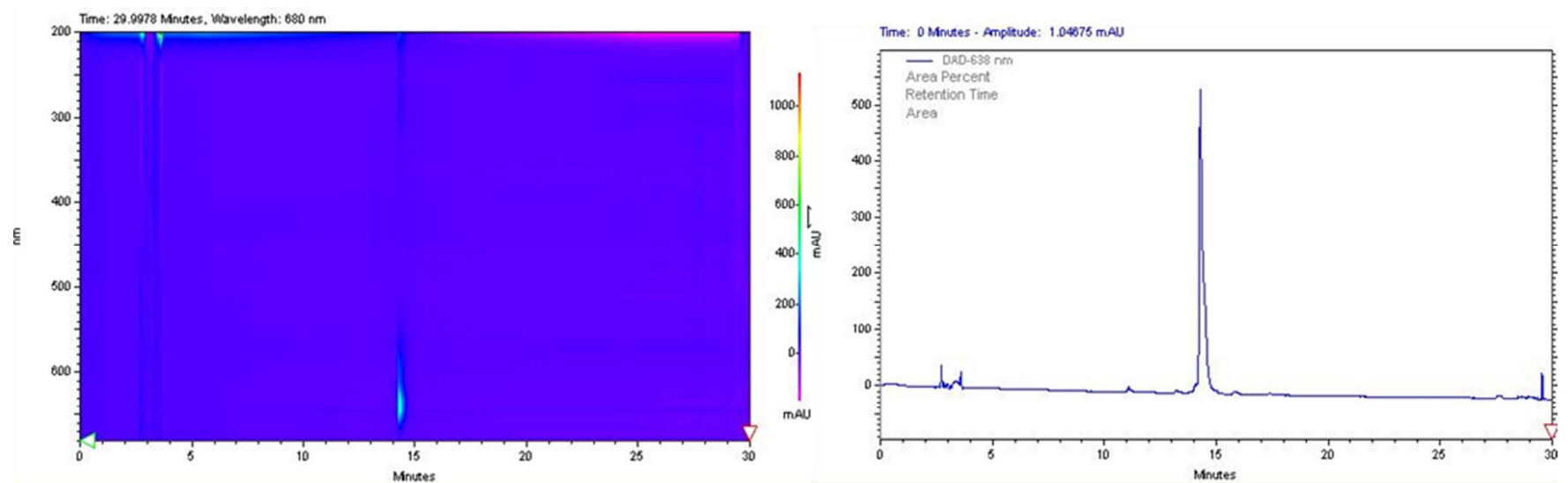

Figure S17. Cy5-Trolox-DBCO: Left: HPLC 3D chromatogram. Right: HPLC 2D chromatogram at $680 \mathrm{~nm}$.

\section{REFERENCES:}

(1) Dedecker, P.; Duwé, S.; Neely, R. K.; Zhang, J. Localizer: Fast, Accurate, Open-Source, and Modular Software Package for Superresolution Microscopy. J. Biomed. Opt. 2012, $17,126008$.

(2) Su, T.; Das, S. K.; Xiao, M.; Purohit, P. K. Transition between Two Regimes Describing Internal Fluctuation of DNA in a Nanochannel. PLoS One 2011, 6, e16890.

(3) Gupta, D.; Sheats, J.; Muralidhar, A.; Miller, J. J.; Huang, D. E.; Mahshid, S.; Dorfman, K. D.; Reisner, W. Mixed Confinement Regimes during Equilibrium Confinement Spectroscopy of DNA. J. Chem. Phys. 2014, 140, 214901. 
(4) Wang, J.; Gao, H. Stretching a Stiff Polymer in a Tube. J. Mater. Sci. 2007, 42, 88388843.

(5) Gunther, K.; Mertig, M.; Seidel, R. Mechanical and Structural Properties of YOYO-1 Complexed DNA. Nucleic Acids Res. 2010, 38, 6526-6532.

(6) BioNano Genomics Website http://www.bionanogenomics.com/.

(7) Reisner, W.; Morton, K. J.; Riehn, R.; Wang, Y. M.; Yu, Z.; Rosen, M.; Sturm, J. C.; Chou, S. Y.; Frey, E.; Austin, R. H. Statics and Dynamics of Single DNA Molecules Confined in Nanochannels. Phys. Rev. Lett. 2005, 94, 196101.

(8) Nyberg, L.; Persson, F.; Akerman, B.; Westerlund, F. Heterogeneous Staining: A Tool for Studies of How Fluorescent Dyes Affect the Physical Properties of DNA. Nucleic Acids Res. 2013, 41, e184-e184.

(9) Noble, C.; Nilsson, A. N.; Freitag, C.; Beech, J. P.; Tegenfeldt, J. O.; Ambjörnsson, T. A Fast and Scalable Kymograph Alignment Algorithm for Nanochannel-Based Optical DNA Mappings. PLoS One 2015, 10, e0121905.

(10) Nilsson, A. N.; Emilsson, G.; Nyberg, L. K.; Noble, C.; Stadler, L. S.; Fritzsche, J.; Moore, E. R. B.; Tegenfeldt, J. O.; Ambjornsson, T.; Westerlund, F. Competitive BindingBased Optical DNA Mapping for Fast Identification of Bacteria - Multi-Ligand Transfer Matrix Theory and Experimental Applications on Escherichia Coli. Nucleic Acids Res. 2014, 42, e118.

(11) Anantharaman, T.; Mishra, B. False Positives in Genomic Map Assembly and Sequence Validation. In Algorithms in Bioinformatics: First International Workshop, WABI 2001, 
Aarhus, Denmark, August 28-31, 2001, Proceedings; Gascuel, O.; Moret, B. M. E., Eds.; Springer Berlin Heidelberg: Berlin, Heidelberg, 2001; pp. 27-40.

(12) Rodgers, J. L.; Nicewander, W. A. Thirteen Ways to Look at the Correlation Coefficient. Am. Stat. 1988, 42, 59-66.

(13) Barlow, R. J. Statistics: A Guide to the Use of Statistical Methods in the Physical Sciences; John Wiley \& Sons, 1989. 